\title{
miR-107 inhibits CDK6 expression, differentiation, and lipid storage in human adipocytes
}

\author{
Maria A. Ahonen ${ }^{a}$, P.A. Nidhina Haridas ${ }^{\mathrm{a}}$, Raghavendra Mysore ${ }^{\mathrm{a}}$, Martin Wabitsch ${ }^{\mathrm{c}}$, \\ Pamela Fischer-Posovszky ${ }^{\mathrm{c}}$, Vesa M. Olkkonen ${ }^{\mathrm{a}, \mathrm{b}, *}$ \\ ${ }^{a}$ Minerva Foundation Institute for Medical Research, Biomedicum 2U, Helsinki, Finland \\ ${ }^{\mathrm{b}}$ Department of Anatomy, Faculty of Medicine, University of Helsinki, Finland \\ ${ }^{c}$ Division of Pediatric Endocrinology and Diabetes, Department of Pediatrics and Adolescent Medicine, University Medical Center Ulm, Ulm, Germany
}

\section{A R T I C L E I N F O}

\section{Keywords:}

miR-107

Notch

CDK6

Adipogenesis

Triglyceride

Adipocyte

\begin{abstract}
A B S T R A C T
MicroRNA-107 (miR-107) plays a regulatory role in obesity and insulin resistance, but the mechanisms of its function in adipocytes have not been elucidated in detail. Here we show that overexpression of miR-107 in preand mature human Simpson-Golabi-Behmel syndrome (SGBS) adipocytes attenuates differentiation and lipid accumulation. Our results suggest that miR-107 controls adipocyte differentiation via CDK6 and Notch signaling. CDK6 is a validated target of miR-107 and was downregulated upon miR-107 overexpression. Notch3, a signaling receptor involved in adipocyte differentiation, has been shown to decrease upon CDK6 depletion; Here Notch3 and its target Hes1 were downregulated by miR-107 overexpression. In mature adipocytes, miR-107 induces a triglyceride storage defect by impairing glucose uptake and triglyceride synthesis. To conclude, our data suggests that miR-107 has distinct functional roles in preadipocytes and mature adipocytes; Its elevated expression at these different stages of adipocytes may on one hand dampen adipogenesis, and on the other, promote ectopic fatty acid accumulation and reduced glucose tolerance.
\end{abstract}

\section{Introduction}

Adipocytes execute a major function as regulators of energy metabolism, and the primary function of adipose tissue is to store triglycerides (TG) (Ali et al., 2013). However, adipose tissue is also considered as a bona fide endocrine organ due to its ability to secrete a plethora of signaling molecules including cytokines and chemokines that regulate appetite, lipid mobilization and inflammation (Lefterova and Lazar, 2009). Storage of excess energy in the form of TG leads to adipocyte hypertrophy and hyperplasia, causing an increase in adipose tissue mass and adverse metabolic effects (Rosen and Spiegelman, 2006). On the other hand, a defect in adipocyte lipid storage results in ectopic storage of fat in liver or muscles leading to insulin resistance or non-alcoholic fatty liver disease (Simha and Garg, 2006). Adipocyte differentiation and lipid accumulation are regulated by distinct signaling pathways and transcriptional control cascades. The key transcription factors that regulate adipogenesis are CAAT/enhancer-binding protein (C/EBP), and peroxisome proliferator activated receptor $\gamma$ (PPAR- $\gamma$ ). Insulin, mTOR, Notch and Wnt signaling are known to play pivotal roles in regulating adipogenesis (Rosen and MacDougald, 2006; Ross et al., 2004). Studies show that microRNAs (miRNAs) are important regulators of both adipocyte differentiation and metabolism (Rottiers and Näär, 2012; Bartel, 2004; Maurizi et al., 2018).

miRNAs are 19-23 nt long non-coding RNAs. They are significantly involved in the pathogenesis of a plethora of diseases such as cancers, cardiovascular diseases, diabetes, neurodegenerative disorders and epilepsy (Thum et al., 2008; Hwang et al., 2013; Karnati et al., 2015). The enhanced or impaired production and function of miRNAs underlie changes in metabolic dysregulation during the development of insulin resistance (Guay et al., 2011; Nakanishi et al., 2009). The regulatory effects of miRNAs on adipose tissue metabolism are mediated via regulation of cell differentiation, proliferation, apoptosis and appetite (Chen et al., 2013). miRNAs that have been connected to insulin resistance, diabetes or obesity include miR-21, miR-221/222, miR-146b, miR-33a and -b, miR-103, miR-107, miR-192* and miR-143 (Nakanishi et al., 2009; Xie et al., 2009; Rottiers and Näär, 2012; Kilic et al., 2015; Mysore et al., 2016). miR-378, -326, - 193b, -126, - 26a -and a number of others -regulate adiponectin (AdipoQ), which is an important regulator and marker of adipogenesis (Ishida et al., 2014; Santovito et al., 2014; Belarbi et al., 2015). miR-378 and -326 downregulate AdipoQ expression, while miR-193b, -126 and $-26 a$ enhance its expression. miR-103/107 are known to modulate insulin

\footnotetext{
* Corresponding author. Minerva Foundation Institute for Medical Research, Biomedicum 2U, Helsinki, Finland.

E-mail address: vesa.olkkonen@helsinki.fi (V.M. Olkkonen).
} 
sensitivity in mouse models of obesity and their expression is altered in human fatty liver (Trajkovski et al., 2011). However, the functions of miR-107 in adipogenesis and lipid storage in adipocytes in murine models are thus far inconclusive, and very little data is available on miR-107 function in human adipocytes. A systematic study on the function of miR-107 in preadipocytes and mature adipocytes has not been carried out. Therefore, we characterize in this study the effects of miR-107 on human SGBS adipocyte differentiation and on mature adipocyte metabolism.

\section{Methods}

\subsection{Cell culture and transfections}

To study the regulation of adipogenesis in the presence of miR-107, Simpson-Golabi-Behmel syndrome (SGBS) preadipocytes were cultured according to Fischer-Posovszky et al. (2008). Preadipocytes were transfected for $48 \mathrm{~h}$ and further differentiated for 13 days as described (Wabitsch et al., 2001; Fischer-Posovszky et al., 2008). Transfections were done with $100 \mathrm{nM}$ miRNA - 107 mimic (Qiagen, MSY0000104) or non-targeting siRNA (Qiagen, 1027280) by using RNAiMax ${ }^{\mathrm{TM}}$ reagent (Thermo Fisher Scientific, Waltham, MA). Transfected and differentiated cells were harvested or used for assay at day 0 or day 13 of induction depending on the experiments. Mature SGBS adipocytes were transfected with $200 \mathrm{nM}$ miR-107 mimic or NT on day 13 for $72 \mathrm{~h}$ and further used for experiments.

\subsection{Gene expression analyses}

Expression of genes was analyzed at mRNA level by qPCR and for some genes at protein level by western blotting. SGBS cells were cultured and transfected as specified above, after which RNA was isolated from the cells with Purelink ${ }^{\oplus}$ RNA Mini Kit or miRNeasy ${ }^{\circ}$ mini kit (QIAGEN, Gaithersburg, MD), according to the manufacturer's protocol. cDNA was synthesized from RNA with SuperScript ${ }^{\circ}$ VILO $^{\text {TM }}$ synthesis Kit (Invitrogen, Carlsbad, CA). Gene expression analyses were done by qPCR using Roche SYBR-Green ${ }^{\circledR}$ master mix and a LightCycler 480 II Real-Time PCR system (Roche Applied Science, Penzberg, Germany). Crossing point $(\mathrm{Cp})$ value was calculated from each amplification curve, which was normalized to $\mathrm{Cp}$ values of housekeeping genes succinate dehydrogenase complex subunit A (SDHA) and actin. Primer sequences are specified in Table 1.

For western blotting cells were lysed with RIPA buffer (buffer tris$\mathrm{HCl} 15 \mathrm{mM}$, pH 7.4 containing NP40 1\%, sodium deoxycholate $1.25 \%$, $\mathrm{NaCl} 150 \mathrm{mM}$, EDTA $1 \mathrm{mM}$ and 1\% SDS) and equal amounts of protein were loaded on 10 or $12 \%$ sodium dodecyl sulfate polyacrylamide gel electrophoresis (SDS-PAGE) (Fast Cast TGX Stain-free) gels. Blotting on PVDF membranes was carried out with BioRad Transblot system. After blocking for unspecific antibody binding, blots were probed overnight with anti-AdipoQ (Sigma A6354), anti-Glut4 (Cell Signaling Technology 2213), anti-Notch3 (Cell Signaling Technology 2889), antiCDK6 (Novus Biologicals NBP1-87262), anti-Hes1 (Santa Cruz 25392), anti-p-Rb (Cell Signaling Technology 9307) and anti-PLIN2 (Thermo Scientific PA1-46158) in 5\% milk in TBS with $0.1 \%$ Tween. Signals were detected with enhanced chemiluminescence (ECL) (Thermo Scientific, Waltham, MA or BioRad Clarity $\mathrm{Max}^{\mathrm{TM}}$ ) using BioRad Chemidoc imaging system. Finally, the signal was quantified with Image Lab by normalizing the band signal to the total protein intensity.

\subsection{Lipid droplet staining and triglyceride assay}

Transfected SGBS cells on coverslips were washed and fixed with 4\% paraformaldehyde in phosphate-buffered saline (PBS). Cells were
Table 1

Sequences of primers used for qPCR analyses.

\begin{tabular}{ll}
\hline Gene & Primer sequence \\
\hline DGAT1 Forward & GGTCCCCAATCACCTCATCTG \\
DGAT1 Reverse & TGCACAGGGATGTTCCAGTTC \\
DGAT2 Forward & ATTGCTGGCTCATCGCTGT \\
DGAT2 Reverse & GGGAAAGTAGTCTCGAAAGTAGC \\
AP2 Reverse & ACACTGATGATCATGTTAGGTTTGG \\
AP2 Forward & GCTTTTGTAGGTACCTGGAAACTT \\
GLUT4 Reverse & CAGGTGAGTGGGAGCAATC \\
GLUT4 Forward & ATCCTTGGACGATTCCTCATTGG \\
AdipoQ Reverse & TACTCCGGTTTCACCGATGTC \\
AdipoQ Forward & TGCTGGGAGCTGTTCTACTG \\
LPL Reverse & GGCCACAAGTTTTGGCACC \\
LPL Forward & ACAAGAGAGAACCAGACTCCAA \\
FAS Forward & AACTCCAAGGACACAGTCACC \\
FAS Reverse & CAGCTGCTCCACGAACTCAA \\
CD36 Forward & AAGCCAGGTATTGCAGTTCTTT \\
CD36 Reverse & GCATTTGCTGATGTCTAGCACA \\
PLIN2 Forward & TTGCAGTTGCCAATACCTATGC \\
PLIN2 Reverse & CCAGTCACAGTAGTCGTCACA \\
PPAR $r$ Reverse & GAGGGAGTTGGAAGGCTCTTC \\
PPAR Forward & GATCCAGTGGTTGCAGATTACAA \\
IL-6 Forward & GGTACATCCTCGACGGCATCT \\
IL-6 Reverse & GTGCCTCTTTGCTGCTTTCAC \\
SREBP1c Forward & GCAGGGGTAGGGCCAACGGCCT \\
SREBP1c Reverse & CATGTCTTCGAAAGTGCAATCC \\
TNF- $\alpha$ Forward & CCCAGGCAGTCAGATCATCTTC \\
TNF- $\alpha$ Reverse & AGCTGCCCCTCAGTTGA \\
&
\end{tabular}

then permeabilized with $0.1 \%$ Triton X-100 in PBS, after which they were washed thrice and stained with Bodipy 493/503 for 20 minutes at room temperature. The coverslips were mounted with Moviol (Calbiochem, La Jolla, CA) containing $50 \mathrm{mg} / \mathrm{ml}$ 1,4-Diazabicyclo[2.2.2] octane (Sigma-Aldrich) and $5 \mu \mathrm{g} / \mathrm{ml}$ DAPI (Thermo Scientific/ Molecular Probes) to stain the nuclei. Imaging was done with oil 63X objective and Colibri laser (Zeiss, Oberkochen, Germany) with the same exposure time for all samples and images were captured with Zen 2 software (Zeiss). Cell number, area and size of lipid droplets were quantified with Image J. Total triglycerides (TG) in the SGBS adipocytes were quantified using GPO-PAP TG assay kit (Cobas, Roche/Hitachi, Tokyo, Japan). TG levels were normalized to total protein content.

\subsection{Glucose uptake}

Mature SGBS adipocytes were transfected with $200 \mathrm{nM}$ miR-107 or non-targeting control siRNA for $72 \mathrm{~h}$, followed by treatment with $100 \mathrm{nM}$ insulin for $15 \mathrm{~min}$ and incubation with $5 \mu \mathrm{Ci} / \mathrm{ml}\left[{ }^{3} \mathrm{H}\right]$-2-deoxyd-glucose (PerkinElmer, Waltham, MA, USA) and $100 \mu \mathrm{M}$ 2-deoxyglucose for $10 \mathrm{~min}$. Glucose uptake was terminated using ice-cold PBS and the cells were washed with ice-cold PBS for three times. Adipocytes were lysed with $0.5 \%$ SDS and radioactivity was measured by liquid scintillation counting.

\subsection{Palmitic acid incorporation and triglyceride measurements}

Transfected SGBS adipocytes were labeled with $\left[{ }^{3} \mathrm{H}\right]$ palmitic acid (Perkin Elmer) in 2\% BSA containing culture medium for $2 \mathrm{~h}$. The cells were then washed and harvested in $2 \% \mathrm{NaCl}$. Total lipids were extracted according to Bligh and Dyer (1959). Lipid classes were separated on TLC (Merck) using neutral lipid solvent system (hexane 65: diethylether 15: acetic acid: 1 water $0.25 \mathrm{~V} / \mathrm{V}$ ). Triolein was used as marker for TG and diolein for diacylglycerol (DAG). The corresponding bands were scraped and the radioactivity was measured by liquid scintillation counting. The counts were normalized to total protein. 


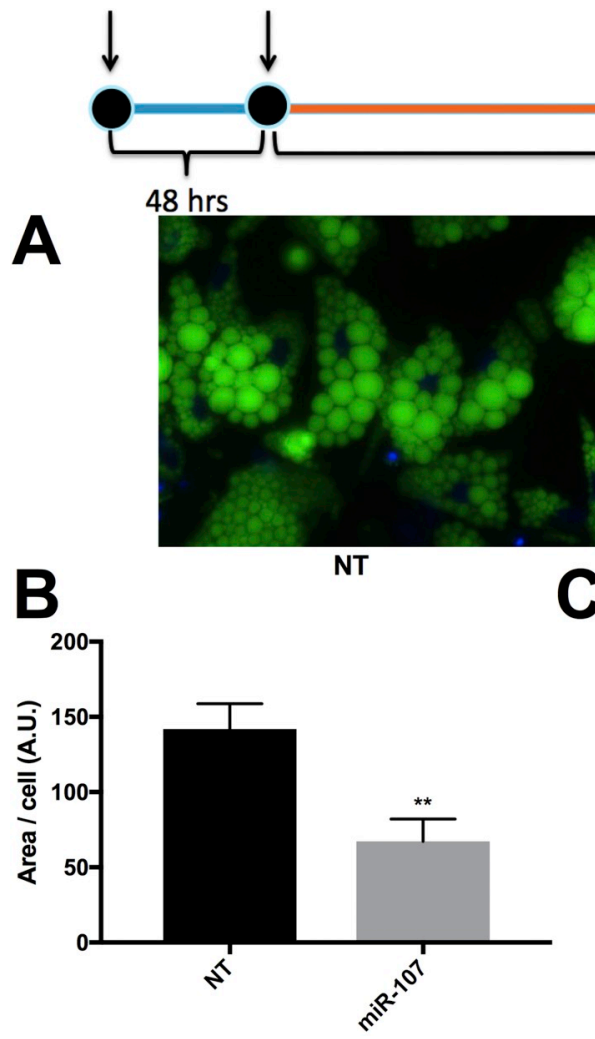

\section{Results}

\section{1. miRNA-107 inhibits adipocyte differentiation in human SGBS} adipocytes

miR-107 is upregulated in obesity and connected to insulin resistance; It is reported to affect adipocyte formation but the results are somewhat inconsistent and the underlying mechanisms have remained unclear. Therefore, we wanted to confirm the effect of miR-107 on human SGBS adipocyte differentiation. We transfected SGBS preadipocytes with miR-107 or non-targeting control (NT) siRNA for $48 \mathrm{~h}$ and further differentiated them for 13 days. miR-107 inhibited the adipocyte differentiation as detected by reduced adipocyte lipid accumulation (Fig. 1A). Total TG in the differentiated adipocytes was significantly reduced by $33 \%$ in the miR-107 transfected cells (Fig. 1C) as compared to NT cells. The miR-107 transfected cells differentiated with very small lipid droplets and the total lipid droplet area was reduced by $53 \%$ in miR-107 transfected cells as compared to the controls (Fig. 1B).

\section{2. miR-107 attenuates the expression of adipocyte differentiation markers}

The inhibition of adipocyte differentiation by miR-107 was confirmed by analyzing the expression of known adipocyte differentiation markers. miR-107 decreased the expression of a mature adipocyte hallmark gene, AdipoQ, both at protein and mRNA levels when the cells were transfected in the preadipocyte stage (Fig. 2A-B). The lipid droplet associated protein perilipin 2 (PLIN2) expression was also significantly reduced at the protein level (Fig. 2B). Likewise, the mRNA expression of several other adipocyte markers (Glucose transporter 4 -GLUT4, diacyl glycerol acyl transferase - DGAT1/2, adipocyte fatty acid binding protein - AP2 and lipoprotein lipase - LPL) was reduced (Fig. 2A) in the miR-107 transfected cells.
Fig. 1. Effect of miR-107 overexpression on triglyceride storage. A) Lipid droplet staining of mature SGBS cells transfected as preadipocytes with nontargeting control siRNA (NT) or miR-107 mimic (miR-107). B) Average lipid droplet area quantified from lipid staining. (Approximately 100 cells in each condition were quantified) C) Triglyceride content on day 13 when transfected as preadipocytes. The timeline of transfection (first arrow) and differentiation (second arrow) is shown at the top. The data represents the mean \pm SEM (Two independent experiments in duplicates). Statistical significance is denoted as $\quad * * p<0.01, \quad * * * p<0.001$ (Mann-Whitney test).

\section{3. miR-107 inhibits CDK6 expression leading to impaired adipocyte differentiation}

CDK6 is a validated target of miR-107 and controls adipocyte differentiation (Sarruf et al., 2005; Varshney et al., 2014). Therefore, it was of interest to analyze whether the miR-107 mediated inhibition of adipogenesis is exerted through CDK6. The expression of CDK6 protein was significantly decreased upon miR-107 transfection of SGBS preadipocytes (Fig. 3A-B). The phosphorylation status of its downstream target retinoblastoma protein $(\mathrm{Rb})$ was significantly decreased in miR107 transfected preadipocytes (Fig. 3A-B).

3.4. Notch3 and its target gene Hes 1 expression is downregulated by miR107 in SGBS preadipocytes

Since Notch3 was recently (Sandel et al., 2018) identified to play a role in adipogenesis and CDK6 is known to regulate Notch3 (Pekkonen et al., 2014), it was of interest to analyze whether the expression of Notch3 and its targets were affected. The expression of Notch3 and its target Hes1 were significantly downregulated in miR-107 transfected preadipocytes compared to NT, as demonstrated by western blotting (Fig. 3A).

\section{5. miR-107 alters lipid storage in mature SGBS adipocytes}

Since miR-107 is increased in the liver of obese human subjects and regulates insulin sensitivity (Trajkovski et al., 2011), we studied whether miR-107 enhances lipid accumulation in mature adipocytes. Mature SGBS adipocytes were transfected with miR-107 or NT and $72 \mathrm{~h}$ later, the total TG content, lipid droplet size and count were measured. Surprisingly, total TG was decreased by $19 \%$ in miR-107 transfected cells (Fig. 4A) and the average lipid droplet size in miR-107 transfected adipocytes was $33 \%$ smaller than in the NT transfected control 


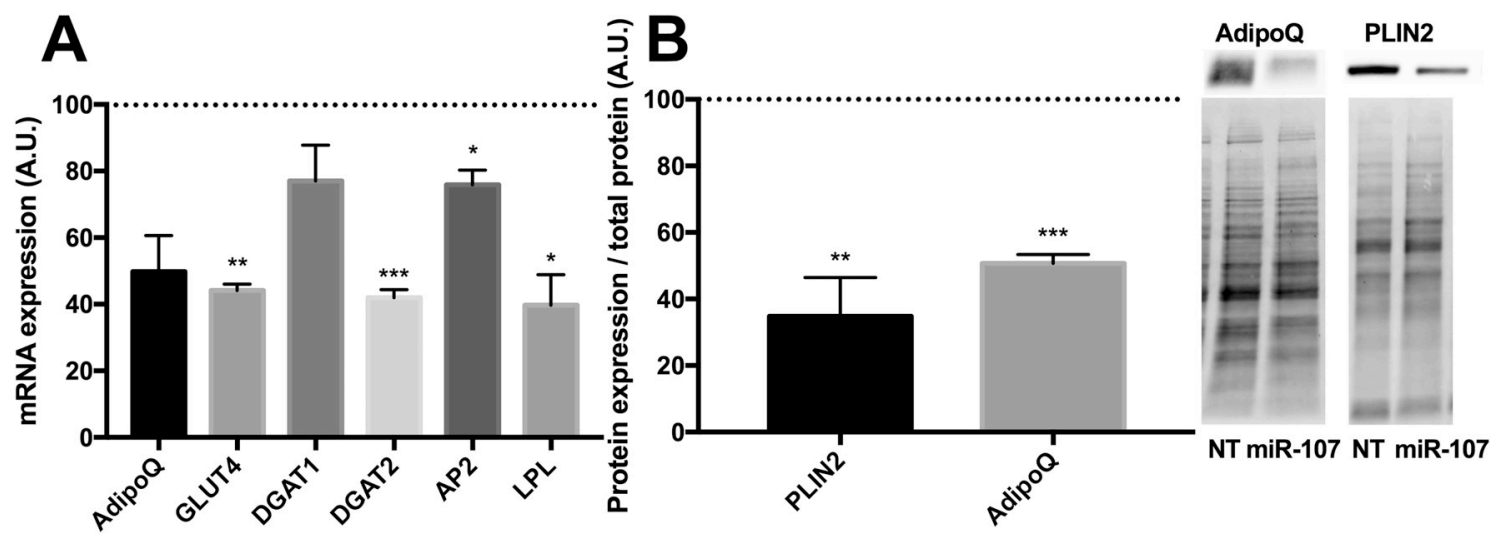

Fig. 2. miR-107 overexpression attenuates adipocyte differentiation. A) mRNA expression of adipocyte differentiation markers after miR-107 transfection. B) Quantification of protein expression of adiponectin (AdipoQ) and perilipin 2 (PLIN2) in miR-107 overexpressing cells. Non-targeting control is indicated by a dashed line. Representative immunoblots are shown on the right side; Top: immunodetected bands; below: total protein of the lanes. The data represents the mean \pm SEM $(\mathrm{n}=4) .{ }^{*} p<0.05,{ }^{* *} p<0.01,{ }^{* * *} p<0.001$ (Student's t-test).

adipocytes (Fig. 4B). However, an increase in the total number of lipid droplets was detected, reflecting an elevated number of small lipid droplets (Fig. 4C). These observations suggest a defect in lipid droplet formation in miR-107 transfected mature adipocytes.

3.6. miR-107 transfected adipocytes exhibit a decrease in glucose uptake, $T G$ synthesis and enhanced inflammatory gene expressions

The defective lipid storage in SGBS mature adipocytes could be due to decreased glucose uptake and TG synthesis. In miR-107 transfected adipocytes compared to NT transfected ones, glucose uptake was significantly reduced by $38 \%$ (Fig. 5A) and palmitic acid (PA) incorporation to TG was significantly decreased by $48 \%$ as compared to NT transfected control cells (Fig. 5C). A decrease in PA incorporation was also observed in diacylglycerols, although it did not reach statistical significance (Fig. 5D). These defects are most likely due to downregulation of multiple genes by miR-107 in the transfected cells. GLUT4 expression was decreased in these cells both at mRNA and protein levels. Moreover, a number of genes related to fatty acid and triglyceride synthesis were downregulated including FAS, DGAT1, DGAT2, CD36, and SREBP1-C (Fig. 5B). The inflammatory markers tumor necrosis growth factor $\alpha$ (TNF- $\alpha$ ) and interleukin 6 (IL-6) (Fig. 5E) were upregulated and AdipoQ expression was reduced at the mRNA level (Fig. 5B). The reduction of AdipoQ at the protein level was not statistically significant, however a decreasing tendency was detectable.

\section{Discussion}

miR-107 is known to regulate insulin sensitivity in mouse models mainly by altering liver metabolism (Trajkovski et al., 2011). The function of adipocyte-expressed miR-107 in adipocyte lipid metabolism and differentiation is poorly understood, and studies in murine cells and animal models are inconclusive. When adipocyte miR-107 was directly injected into mice fat pads, the size of adipocytes increased. On the contrary, rat stromal vascular cells were not able to efficiently differentiate into adipocytes upon miR-107 overexpression (Trajkovski et al., 2011). Therefore, a systematic study was required to identify the effect of miR-107 on adipocyte differentiation and metabolism.

In this study, the effects of miR-107 on adipocyte differentiation were analyzed by transfecting the SGBS fibroblasts (preadipocytes) and further differentiating them. The effects of miR-107 in mature adipocytes were studied by transfecting mature SGBS adipocytes. A defect in adipocyte differentiation and lipid droplet formation were observed in the cells when they were transfected with miR-107 at preadipocyte

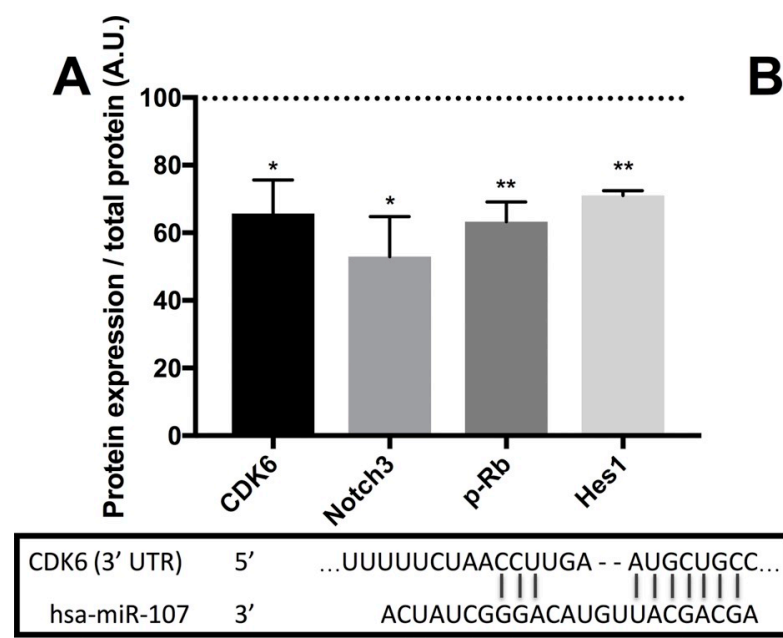

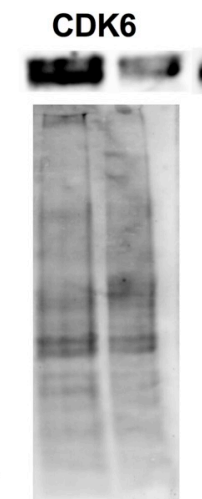

NT miR107

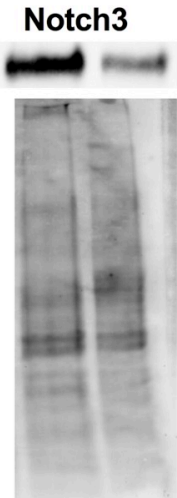

NT miR-

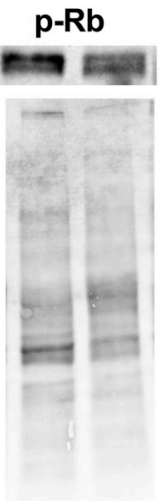

NT miR-

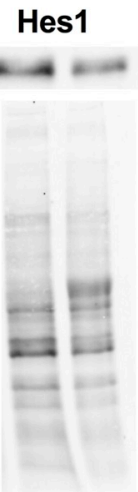

NT miR-

107

Fig. 3. miR-107 inhibits CDK6 expression and downstream signaling. A) Quantification of CDK6, Notch3, p-Rb and Hes1 from western blots of NT or miR-107 transfected preadipocytes. Non-targeting control is indicated by a dashed line. Quantification of western blots and representative western images. B) Top: immunodetected bands; below: total protein of the lanes. The data represents the mean \pm SEM $(\mathrm{n}=4) .{ }^{*} p<0.05,{ }^{*} p<0.01$ (Student's t-test). 
A

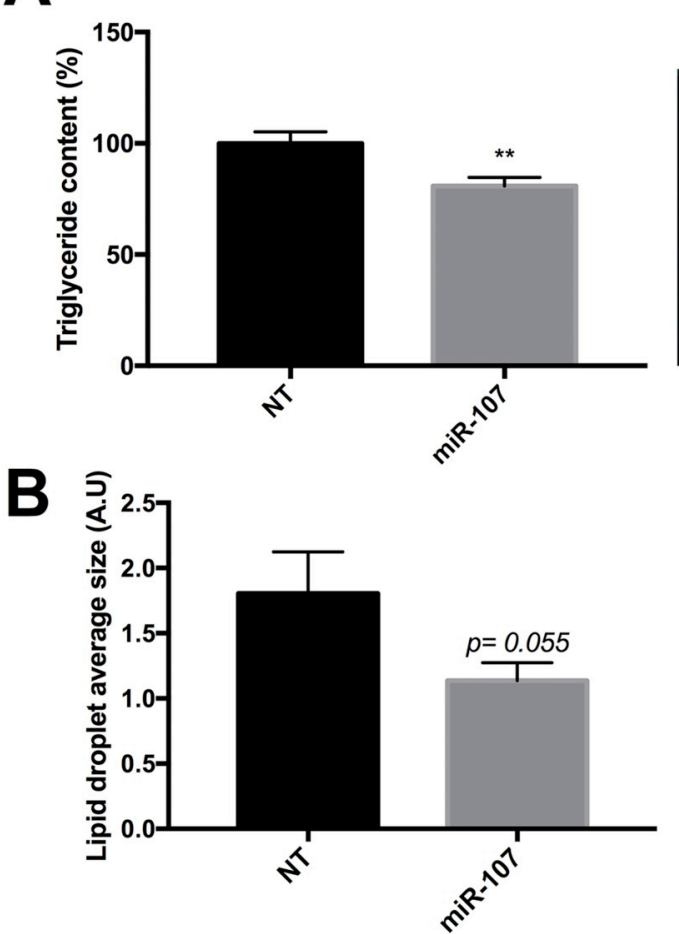

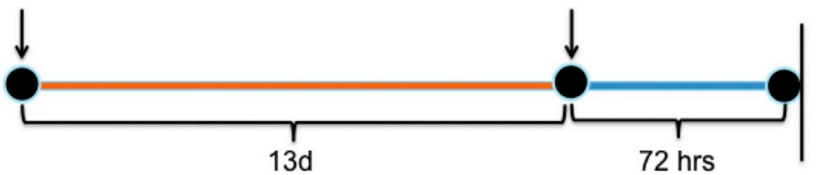

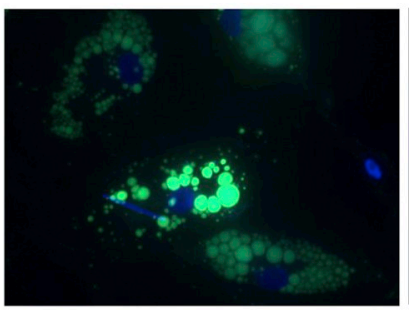

NT

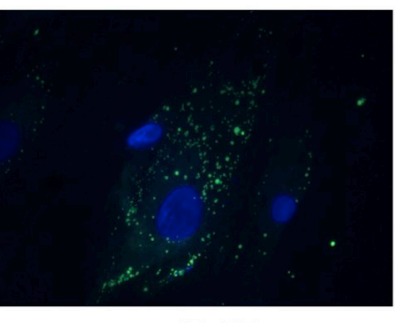

miR-107

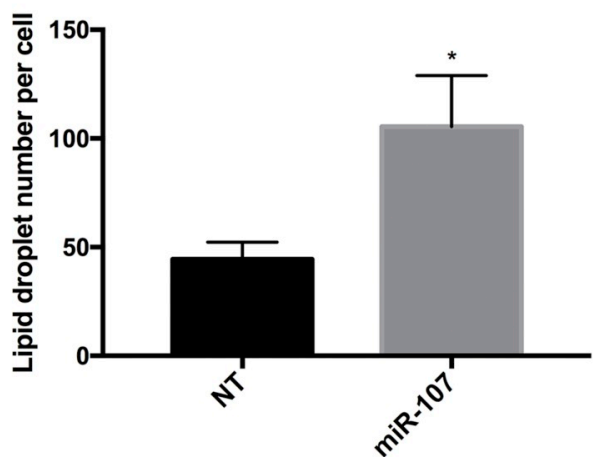

Fig. 4. Triglyceride storage and lipid droplet formation are altered upon miR-107 transfection in mature adipocytes. A) Triglyceride quantification ( $\mathrm{n}=4$ ) and representative lipid droplet staining B) average lipid droplet size and C) number of lipid droplets (200-300 cells in each condition were quantified) in mature adipocytes transfected with NT or miR-107. The timeline of differentiation (first arrow) and transfection (second arrow) is shown at the top. The data represents the mean \pm SEM. Statistical significance is denoted as $* p<0.05,{ }^{* *} p<0.01$ (Mann-Whitney test).

stage. The adipogenic differentiation defect was confirmed by analyzing the expression of differentiation markers like GLUT4, AdipoQ, PLIN2, DGAT1 and -2, LPL, GLUT4, and aP2 at day 13 of differentiation.

Since CDK6 is a validated target of miR-107 (Feng et al., 2012) and is implicated to have a role in adipocyte differentiation, the expression of CDK6 and phosphorylation status of its target Rb were analyzed. Both CDK6 expression and phosphorylation of Rb were affected by miR107 transfection, demonstrating that miR-107 controls CDK6 signaling in preadipocytes. CDK6 has direct targets that affect adipocyte differentiation, such as PPAR $\gamma$, (Sarruf et al., 2005). It also phosphorylates the retinoblastoma protein $(\mathrm{Rb})$, enhancing cultured cells' commitment to S-phase. Therefore, inhibition of CDK6 mediated phosphorylation of $\mathrm{Rb}$ could cause G1 phase arrest (Pekkonen et al., 2014). A cell cycle arrest in the G1 phase has been shown to inhibit differentiation in 3T3L1 adipocytes (Ferguson et al., 2016; Auld and Morrison, 2006; Auld et al., 2007). When CDK6 was silenced, Notch3 and its targets Hes1 and Hey1, were decreased (Pekkonen et al., 2014). Hes1 and Hey1 are known to alter adipocyte differentiation and to have both promoting and inhibitory roles in this process (Ross et al., 2004; Scroyen et al., 2015). In the present human SGBS model, decrease of Hes1 expression indicates a proadipogenic role of Hes1. Thus, CDK6 affects several pathways to promote differentiation (Fig. 6). Notch3 expression is also reported to be upregulated during adipogenesis (Sandel et al., 2018), however, silencing of Notch3 in that study did not decrease adipogenesis. The observed decrease in Notch 3 could thus also be a consequence rather than a cause of the defect in adipogenesis. CDK6 is an emerging therapy target for obesity due to its role in maintaining white adipose phenotype. It is also an inhibitor of white to brown adipose conversion (Hou et al., 2018). This study supports an important role of
CDK6 in adipocyte differentiation. More studies are warranted to clarify in detail the function of CDK6 in Notch3 signaling and adipocyte metabolism.

We further characterized the effect of miR-107 on mature adipocytes. A defect in TG storage most likely mediated by a decrease in glucose uptake and impaired TG synthesis was observed. The adipocytes also displayed an elevated inflammatory status, as indicated by elevated expression of TNF- $\alpha$ and IL- 6 and a reduction of AdipoQ mRNA. Together the data show that the mature adipocytes with an elevated amount of miR-107 have the potential to become insulin resistant, which could promote impaired glucose tolerance in human subjects. Genes involved in fatty acid and glucose transport and TG synthesis were dampened substantiating the phenotype observed. These data show that, depending on the expression of miR-107 in preadipocytes or mature adipocytes, its consequences on obesity might differ. In early stages or transition from overweight to obese, miR-107 might prohibit further adipocyte hypertrophy or generation of adipocytes from preadipocytes leading to reduced weight gain. On the other hand, if miR-107 is elevated in mature adipocytes in obese conditions it might lead to ectopic fatty acid accumulation and reduced glucose tolerance due to the defective glucose uptake and storage of fatty acids in adipocytes. In NAFLD elevated levels of miR-107 are observed in the liver, leading to reduced insulin sensitivity (Trajkovski et al., 2011). miR-107 might thus have a higher impact on human obese subjects with NAFLD than on obese subjects with a normal liver fat content.

In conclusion, miR-107 reduces adipogenesis by downregulating CDK6 and its downstream targets. In mature adipocytes, miR-107 reduced glucose uptake and TG synthesis, suggesting that it may promote ectopic fatty acid accumulation and reduced glucose tolerance. 
A
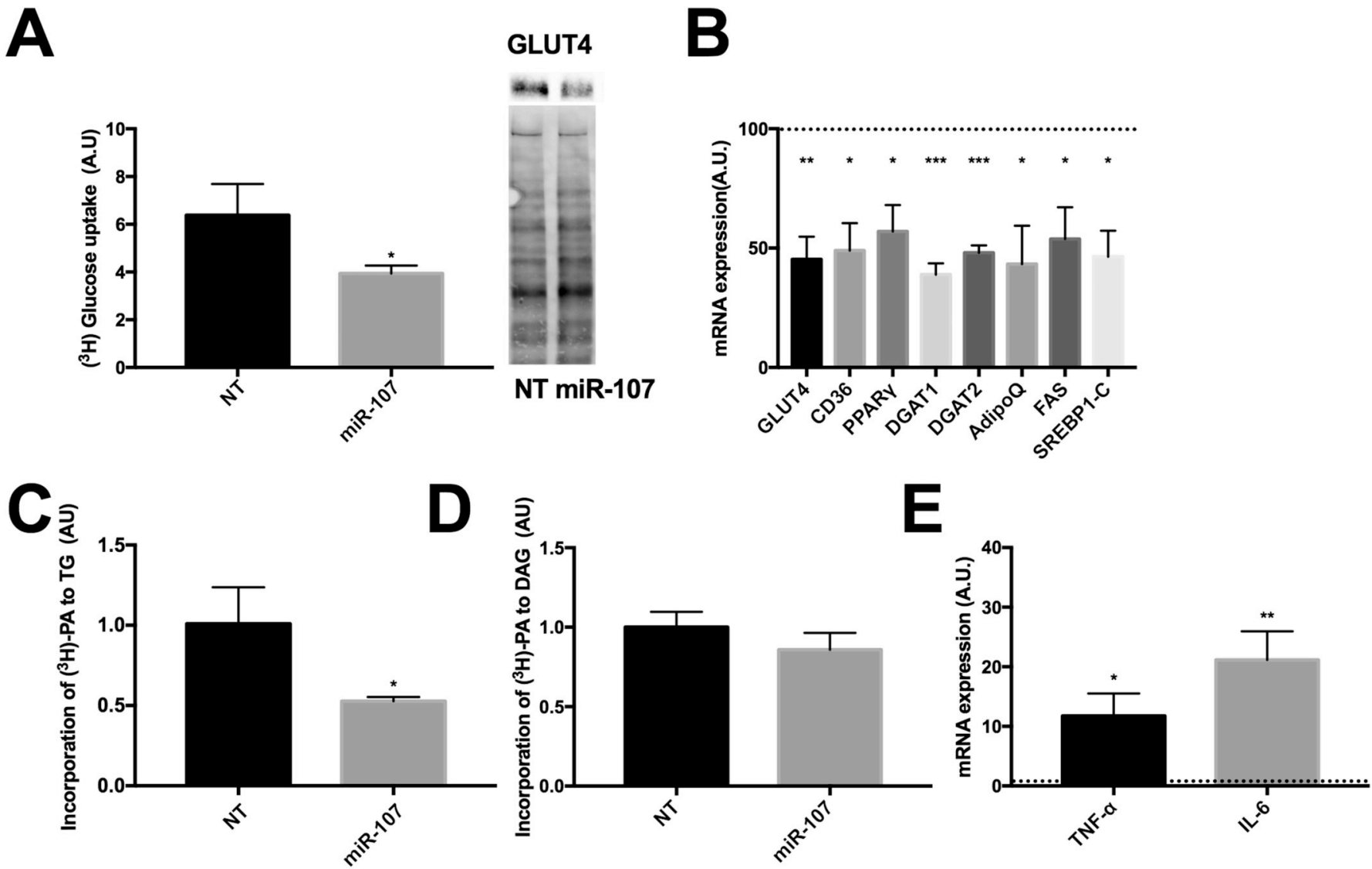

Fig. 5. Glucose and triglyceride metabolism are impaired by miR-107. A) Glucose uptake and GLUT4 protein expression B) mRNA expression of genes involved in glucose and fatty acid transport and triglyceride synthesis, C) Incorporation of palmitic acid into TG and, D) into DAG, E) inflammatory gene expression in NT and miR-107 transfected SGBS adipocytes. Non-targeting control is indicated by a dashed line. The data represents the mean \pm SEM ( $\mathrm{n}=3-4)$. * $p<0.05$, ** $p<0.01$, $* * * p<0.001$ (Mann-Whitney and for gene expression, Student's t-test).

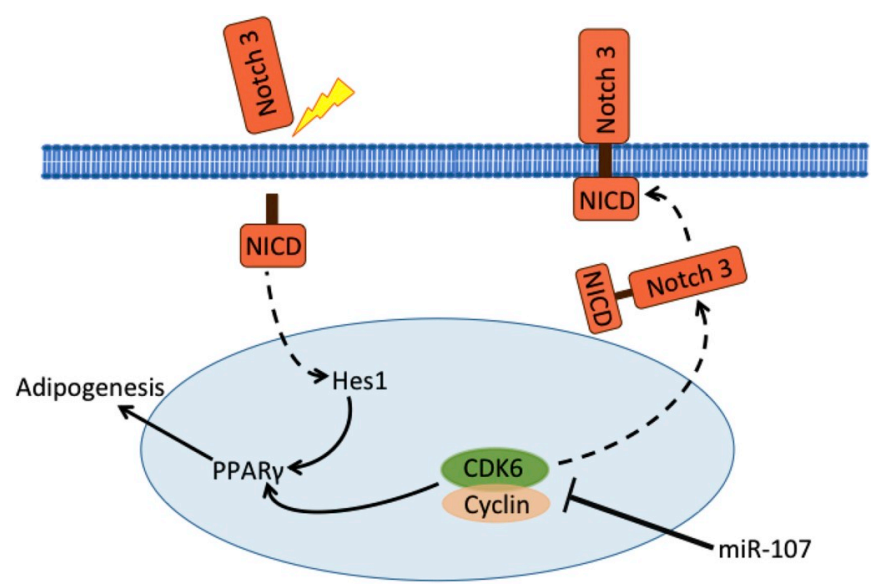

Fig. 6. Schematic representation of the possible mechanism of miR-107 induced inhibition of adipogenesis.

\section{Acknowledgements}

We thank Dr. Marko Hyytiäinen, Prof. Kid Törnquist and Dr. Muhammad Yasir Asghar for kindly providing the antibodies. We thank Jennimari Partanen and Mikko Olkkonen for providing technical support. This study was supported by the Finnish Diabetes Research Foundation (P.A.N.H., V.M.O.), the Liv och Hälsa Foundation, the Finnish Foundation for Cardiovascular Research and Sigrid Juselius
Foundation (V.M.O.). PFP receives funding from Baden-Württemberg Stiftung.

\section{References}

Ali, A.T., Hochfeld, W.E., Myburgh, R., Pepper, M.S., 2013. Adipocyte and adipogenesis. Eur. J. Cell Biol. 92, 229-236. https://doi.org/10.1016/J.EJCB.2013.06.001.

Auld, C.A., Fernandes, K.M., Morrison, R.F., 2007. Skp2-mediated p27(Kip1) degradation during S/G2 phase progression of adipocyte hyperplasia. J. Cell. Physiol. 211, 101-111. https://doi.org/10.1002/jcp.20915.

Auld, C.A., Morrison, R.F., 2006. Evidence for cytosolic p27(Kip1) ubiquitylation and degradation during adipocyte hyperplasia*. Obesity 14, 2136-2144. https://doi.org/ 10.1038/oby.2006.250.

Bartel, D.P., 2004. MicroRNAs: genomics, biogenesis, mechanism, and function. Cell 116, 281-297. https://doi.org/10.1016/S0092-8674(04)00045-5.

Belarbi, Y., Mejhert, N., Lorente-Cebrián, S., Dahlman, I., Arner, P., Rydén, M., Kulyté, A., 2015. MicroRNA-193b controls adiponectin production in human white adipose tissue. J. Clin. Endocrinol. Metab. 100, E1084-E1088. https://doi.org/10.1210/jc. 2015-1530.

Bligh, E.G., Dyer, W.J., 1959. A rapid method of total lipid extraction and purification. Can. J. Biochem. Physiol. 37, 911-917. https://doi.org/10.1139/059-099.

Chen, L., Song, J., Cui, J., Hou, J., Zheng, X., Li, C., Liu, L., 2013. microRNAs regulate adipocyte differentiation. Cell Biol. Int. 37, 533-546. https://doi.org/10.1002/cbin. 10063.

Feng, L., Xie, Y., Zhang, H., Wu, Y., 2012. miR-107 targets cyclin-dependent kinase 6 expression, induces cell cycle G1 arrest and inhibits invasion in gastric cancer cells. Med. Oncol. 29, 856-863. https://doi.org/10.1007/s12032-011-9823-1.

Ferguson, B.S., Nam, H., Morrison, R.F., 2016. Curcumin inhibits 3T3-L1 preadipocyte proliferation by mechanisms involving post-transcriptional p27 regulation. Biochem. Biophys. Reports 5, 16-21. https://doi.org/10.1016/j.bbrep.2015.11.014.

Fischer-Posovszky, P., Newell, F.S., Wabitsch, M., Tornqvist, H.E., 2008. Human SGBS cells - a unique tool for studies of human fat cell biology. Obes. Facts. 1, 184-189. https://doi.org/10.1159/000145784.

Guay, C., Roggli, E., Nesca, V., Jacovetti, C., Regazzi, R., 2011. Diabetes mellitus, a microRNA-related disease? Transl. Res. 157, 253-264. https://doi.org/10.1016/j. 
trs1.2011.01.009.

Hou, X., Zhang, Y., Li, W., Hu, A.J., Luo, C., Zhou, W., Hu, J.K., Daniele, S.G., Wang, J., Sheng, J., Fan, Y., Greenberg, A.S., Farmer, S.R., Hu, M.G., 2018. CDK6 inhibits white to beige fat transition by suppressing RUNX1. Nat. Commun. 9, 1023. https://doi. org/10.1038/s41467-018-03451-1.

Hwang, M.S., Yu, N., Stinson, S.Y., Yue, P., Newman, R.J., Allan, B.B., Dornan, D., 2013. miR-221/222 targets adiponectin receptor 1 to promote the epithelial-to-mesenchymal transition in breast cancer. PLoS One 8, e66502. https://doi.org/10.1371/ journal.pone.0066502.

Ishida, M., Shimabukuro, M., Yagi, S., Nishimoto, S., Kozuka, C., Fukuda, D., Soeki, T., Masuzaki, H., Tsutsui, M., Sata, M., 2014. MicroRNA-378 regulates adiponectin expression in adipose tissue: a new plausible mechanism. PLoS One 9, e111537. https://doi.org/10.1371/journal.pone.0111537.

Karnati, H.K., Panigrahi, M.K., Gutti, R.K., Greig, N.H., Tamargo, I.A., 2015. miRNAs: key players in neurodegenerative disorders and epilepsy. J. Alzheim. Dis. 48, 563-580. https://doi.org/10.3233/JAD-150395.

Kilic, I.D., Dodurga, Y., Uludag, B., Alihanoglu, Y.I., Yildiz, B.S., Enli, Y., Secme, M., Bostanc, H.E., 2015. microRNA -143 and -223 in obesity. Gene 560, 140-142. https://doi.org/10.1016/j.gene.2015.01.048.

Lefterova, M.I., Lazar, M.A., 2009. New developments in adipogenesis. Trends Endocrinol. Metabol. 20, 107-114. https://doi.org/10.1016/J.TEM.2008.11.005.

Maurizi, G., Babini, L., Della Guardia, L., 2018. Potential role of microRNAs in the reg ulation of adipocytes liposecretion and adipose tissue physiology. J. Cell. Physiol. https://doi.org/10.1002/jcp.26523. (Epub ahead of print).

Mysore, R., Zhou, Y., Sädevirta, S., Savolainen-Peltonen, H., Nidhina Haridas, P.A., Soronen, J., Leivonen, M., Sarin, A.-P., Fischer-Posovszky, P., Wabitsch, M., YkiJärvinen, H., Olkkonen, V.M., 2016. MicroRNA-192* impairs adipocyte triglyceride storage. Biochim. Biophys. Acta Mol. Cell Biol. Lipids 1861, 342-351. https://doi. org/10.1016/j.bbalip.2015.12.019.

Nakanishi, N., Nakagawa, Y., Tokushige, N., Aoki, N., Matsuzaka, T., Ishii, K., Yahagi, N., Kobayashi, K., Yatoh, S., Takahashi, A., Suzuki, H., Urayama, O., Yamada, N., Shimano, H., 2009. The up-regulation of microRNA-335 is associated with lipid metabolism in liver and white adipose tissue of genetically obese mice. Biochem. Biophys. Res. Commun. 385, 492-496. https://doi.org/10.1016/j.bbrc.2009.05.058.

Pekkonen, P., Järviluoma, A., Zinovkina, N., Cvrljevic, A., Prakash, S., Westermarck, J., Evan, G.I., Cesarman, E., Verschuren, E.W., Ojala, P.M., 2014. KSHV viral cyclin interferes with T-cell development and induces lymphoma through Cdk6 and Notch activation in vivo. Cell Cycle 13, 3670-3684. https://doi.org/10.4161/15384101. 2014.964118

Rosen, E.D., MacDougald, O.A., 2006. Adipocyte differentiation from the inside out. Nat. Rev. Mol. Cell Biol. 7, 885-896. https://doi.org/10.1038/nrm2066.

Rosen, E.D., Spiegelman, B.M., 2006. Adipocytes as regulators of energy balance and glucose homeostasis. Nature 444, 847-853. https://doi.org/10.1038/nature05483.
Ross, D.A., Rao, P.K., Kadesch, T., 2004. Dual roles for the Notch target gene Hes-1 in the differentiation of 3T3-L1 preadipocytes. Mol. Cell Biol. 24, 3505-3513.

Rottiers, V., Näär, A.M., 2012a. MicroRNAs in metabolism and metabolic disorders. Nat. Rev. Mol. Cell Biol. 13, 239-250. https://doi.org/10.1038/nrm3313.

Sandel, D.A., Liu, M., Ogbonnaya, N., Newman, J.J., 2018. Notch3 is involved in adipogenesis of human adipose-derived stromal/stem cells. Biochimie 150, 31-36. https://doi.org/10.1016/j.biochi.2018.04.020.

Santovito, D., De Nardis, V., Marcantonio, P., Mandolini, C., Paganelli, C., Vitale, E., Buttitta, F., Bucci, M., Mezzetti, A., Consoli, A., Cipollone, F., 2014. Plasma exosome MicroRNA profiling unravels a new potential modulator of adiponectin pathway in diabetes: effect of glycemic control. J. Clin. Endocrinol. Metab. 99, E1681-E1685. https://doi.org/10.1210/jc.2013-3843.

Sarruf, D.A., Iankova, I., Abella, A., Assou, S., Miard, S., Fajas, L., 2005. Cyclin D3 promotes adipogenesis through activation of peroxisome proliferator-activated receptor gamma. Mol. Cell Biol. 25, 9985-9995. https://doi.org/10.1128/MCB.25.22.99859995.2005.

Scroyen, I., Bauters, D., Vranckx, C., Lijnen, H.R., 2015. The anti-adipogenic potential of COUP-TFII is mediated by downregulation of the Notch target gene Hey1. PLoS One 10, e0145608. https://doi.org/10.1371/journal.pone.0145608.

Simha, V., Garg, A., 2006. Lipodystrophy: lessons in lipid and energy metabolism. Curr. Opin. Lipidol. 17, 162-169. https://doi.org/10.1097/01.mol.0000217898.52197.18.

Thum, T., Gross, C., Fiedler, J., Fischer, T., Kissler, S., Bussen, M., Galuppo, P., Just, S., Rottbauer, W., Frantz, S., Castoldi, M., Soutschek, J., Koteliansky, V., Rosenwald, A., Basson, M.A., Licht, J.D., Pena, J.T.R., Rouhanifard, S.H., Muckenthaler, M.U., Tuschl, T., Martin, G.R., Bauersachs, J., Engelhardt, S., 2008. MicroRNA-21 contributes to myocardial disease by stimulating MAP kinase signalling in fibroblasts. Nature 456, 980-984. https://doi.org/10.1038/nature07511.

Trajkovski, M., Hausser, J., Soutschek, J., Bhat, B., Akin, A., Zavolan, M., Heim, M.H., Stoffel, M., 2011. MicroRNAs 103 and 107 regulate insulin sensitivity. Nature 474. https://doi.org/10.1038/nature10112.

Varshney, S., Shankar, K., Beg, M., Balaramnavar, V.M., Mishra, S.K., Jagdale, P., Srivastava, S., Chhonker, Y.S., Lakshmi, V., Chaudhari, B.P., Bhatta, R.S., Saxena, A.K., Gaikwad, A.N., 2014. Rohitukine inhibits in vitro adipogenesis arresting mitotic clonal expansion and improves dyslipidemia in vivo. J. Lipid Res. 55, 1019-1032. https://doi.org/10.1194/jlr.M039925.

Wabitsch, M., Brenner, R.E., Melzner, I., Braun, M., Möller, P., Heinze, E., Debatin, K.M., Hauner, H., 2001. Characterization of a human preadipocyte cell strain with high capacity for adipose differentiation. Int. J. Obes. Relat. Metab. Disord. 25, 563-580. https://doi.org/10.10.1038/sj.ijo.0801520.

Xie, H., Lim, B., Lodish, H.F., 2009. MicroRNAs induced during adipogenesis that accelerate fat cell development are downregulated in obesity. Diabetes 58, 1050-1057. https://doi.org/10.2337/db08-1299. 